\title{
ARTICLE OPEN Quantum transport characteristics of heavily doped bismuth selenide nanoribbons
}

\author{
Hao Tang ${ }^{1,2}$, Xuejun Yan $^{3}$, Yucheng Xiong ${ }^{1}$, Kunpeng Dou ${ }^{4}$, Yang Zhao ${ }^{5}$, Jiansheng Jie ${ }^{6}$, Xiaomeng Wang ${ }^{1}$, Qiang Fu ${ }^{1}$, Juekuan Yang ${ }^{5}$, \\ Minghui Lu (iD ${ }^{3}$ and Dongyan $\mathrm{Xu}$ (iD)
}

This work experimentally investigated quantum transport characteristics of heavily doped bismuth selenide topological insulator nanoribbons to understand their physical origins. Transport properties of nanoribbons were measured via a suspended microdevice for eliminating the substrate effect. A series of quantum transport behaviors such as weak antilocalization, Shubnikov-de Haas oscillations, universal conductance fluctuation, and linear perpendicular-field magnetoresistance have been systematically studied to achieve a coherent understanding on their origins in topologically protected surface states, band bending, or bulk states. The parallel-field magnetoresistance, however, is found to be diverse, which can exhibit negative or positive values for the whole measurement range of the magnetic field strength or change from positive to negative values with the increase of the magnetic field strength. The tunable behavior of the parallel-field magnetoresistance is suggested to be the collective effects of the positive magnetoresistance from surface transport and the negative magnetoresistance possibly owing to the axial anomaly, resulting from long-range ionic impurity-scattering processes in bulk carriers.

npj Quantum Materials (2019)4:1 ; https://doi.org/10.1038/s41535-018-0142-z

\section{INTRODUCTION}

As a new quantum state of matter, the three-dimensional (3D) topological insulators (TIs) are fully gapped in the bulk but exhibit an odd number of massless Dirac cones on the surface, originating from the strong spin-orbit interaction that causes a band inversion. ${ }^{1}$ Electrons in these topological surface states (TSSs) have a helical spin structure in momentum space that is protected by time-reversal symmetry (TRS), which can be applied in spintronics and quantum computing. ${ }^{2,3}$ To date, despite the success of photoemission ${ }^{4}$ and scanning tunneling spectroscopy ${ }^{5}$ in identifying chiral surface states, most efforts have been devoted to the study of quantum transport behaviors of $\mathrm{Tls}^{6-13}$ which is necessary to understand and make the best use of electronic properties of Dirac electrons for various applications.

To access the Dirac cone for electron transport, the chemical potential must lie in the bulk band gap and the bulk carrier density should be reduced to zero. ${ }^{14}$ However, all the existing $\mathrm{TI}$ materials studied to date are not true Mott insulators. Interstitials, vacancies, and antisite doping are common issues that give rise to a residual bulk carrier density. 415 The problem can be partially mitigated by optimizing growth conditions for high-quality crystallinity, $^{10}$ doping, ${ }^{4,16}$ increasing the surface-to-volume ratio, ${ }^{9,17}$ or controlling the Fermi energy via a gate bias, ${ }^{13}$ however, the produced Tls, at best, are weakly insulating bad metals that cannot exhibit the pure surface state transport. Encouragingly, some signatures of two-dimensional (2D) surface transport have already been observed such as Shubnikov-de Haas oscillations (SdHOs), ${ }^{6,7}$ weak antilocalization (WAL), ${ }^{8,9}$ nonsaturating linear magnetoresistance (LMR), ${ }^{10,11}$ and universal conductance fluctuation (UCF). ${ }^{12,13}$ Nevertheless, the quantum signals of electron transport of TSSs are often obscured by electron transport in parallel conduction channels including both the bulk and the $2 \mathrm{D}$ electron gas (2DEG) formed near the surface owing to bending of bulk bands. Both the bulk transport and the 2DEG transport complicate the observed phenomena and lead to the widely varying data in the literature, thus resulting in controversial and conflicting analyses and interpretations. ${ }^{15,18-20}$ Few researchers have systematically studied and discussed multiple quantum signatures in one material system.

In this work, we report a detailed transport study of Sb-Tecodoped bismuth selenide $\left(\mathrm{Bi}_{2} \mathrm{Se}_{3}\right) \mathrm{TI}$ nanoribbons. Magnetoresistance (MR) measurements were performed via a suspended microdevice to prevent the substrate effect. The quantum transport behaviors including WAL, SdHOs, and UCFs are systematically investigated and comprehensively discussed in heavily doped $\mathrm{Bi}_{2} \mathrm{Se}_{3} \mathrm{TI}$ nanoribbons. Anisotropic MR phenomena are observed, which depend on the direction of the magnetic field relative to the electric field. Unlike the $L M R$ for $E \perp B(E$ and $B$ denote the electric and magnetic fields, respectively) that exists in all samples, the parallel-field $\left(E_{\|} B\right) M R$ varies from sample to sample. This tunable parallel-field $M R$ is considered to be the collective effects of a positive MR from surface transport and a negative MR possibly from bulk transport due to ionic impurity scattering.

\footnotetext{
${ }^{1}$ Department of Mechanical and Automation Engineering, The Chinese University of Hong Kong, Shatin, New Territories, Hong Kong Special Administrative Region, People's Republic of China; ${ }^{2}$ International Demonstration School of Materials Science \& Engineering, State Key Laboratory of Advanced Technology for Materials Synthesis and Processing, Wuhan University of Technology, Wuhan 430070, People's Republic of China; ${ }^{3}$ Department of Materials Science and Engineering, Nanjing University, Nanjing 210093, People's

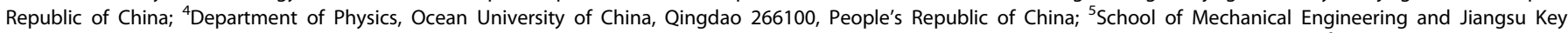
Laboratory for Design and Manufacture of Micro-Nano Biomedical Instruments, Southeast University, Nanjing 210096, People's Republic of China and ${ }^{6}$ Jiangsu Key Laboratory for Carbon-Based Functional Materials \& Devices, Institute of Functional Nano \& Soft Materials (FUNSOM), Soochow University, Suzhou 215123, People's Republic of China Correspondence: Juekuan Yang (yangjk@seu.edu.cn) or Minghui Lu (luminghui@nju.edu.cn) or Dongyan Xu (dyxu@mae.cuhk.edu.hk)
}

Received: 15 July 2018 Accepted: 6 December 2018

Published online: 03 January 2019 

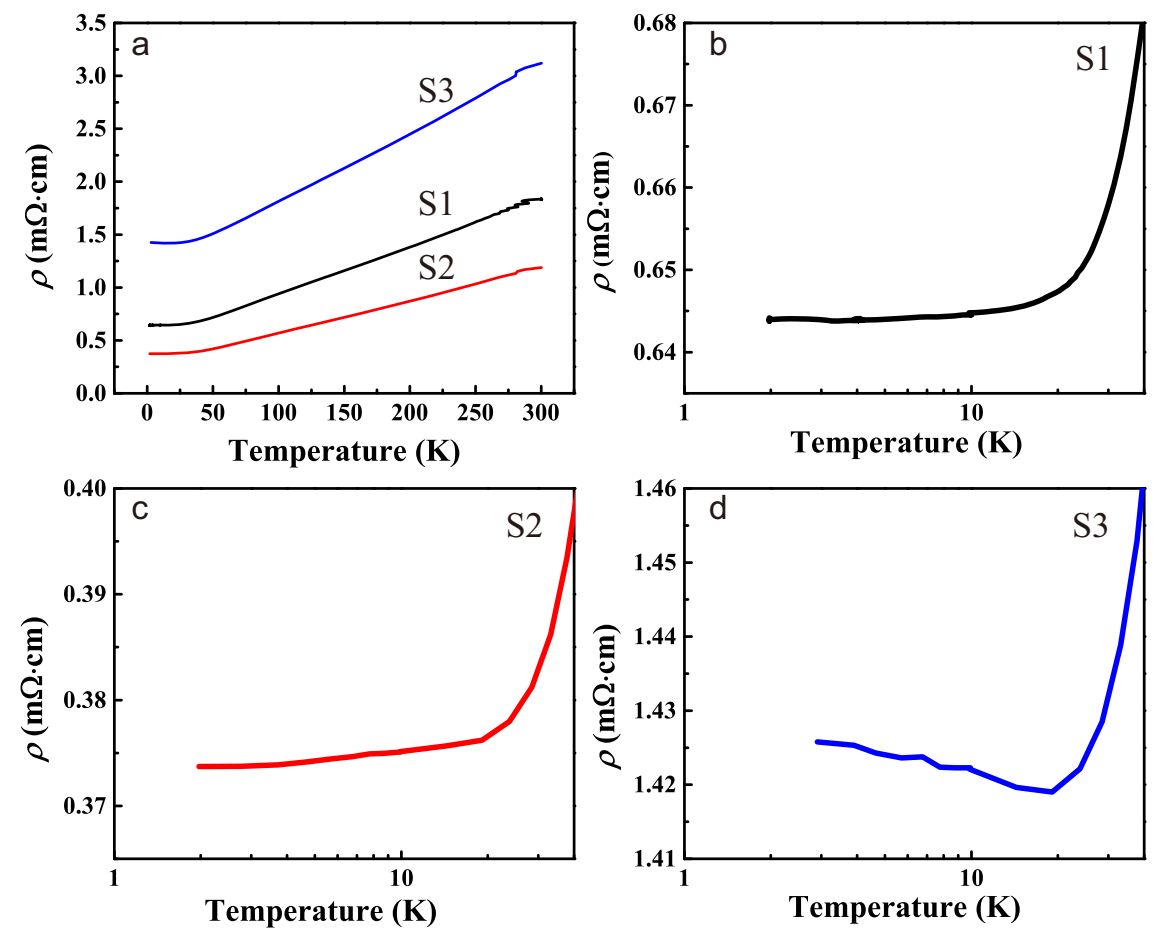

Fig. 1 a Temperature-dependent resistivity of three $\mathrm{Bi}_{2} \mathrm{Se}_{3}$ nanoribbons ( $\mathrm{S} 1, \mathrm{~S} 2$, and $\mathrm{S} 3$ ). b-d Resistivity of $\mathrm{S} 1, \mathrm{~S} 2$, and $\mathrm{S} 3$ in the low temperature range

Table 1. The thickness $(t)$, width $(w)$, suspended length $(L)$, atomic ratios, carrier density $(n)$, and mobility $(\mu)$ of three measured nanoribbons (S1, S2, and S3)

\begin{tabular}{lllllll}
\hline Sample & $t$ & $\begin{array}{l}w \\
(\mathrm{~nm})\end{array}$ & $\begin{array}{l}\mathrm{(nm}) \\
(\mu \mathrm{m})\end{array}$ & $\begin{array}{l}\text { Atomic ratios } \\
\text { Bi: Se: Sb: Te }\end{array}$ & $\begin{array}{l}n \\
\left(\mathrm{~cm}^{-3}\right)\end{array}$ & $\begin{array}{l}\mu \\
\left(\mathrm{cm}^{2} / \mathrm{V} \cdot \mathrm{s}\right)\end{array}$ \\
\hline S1 & 76 & 548 & 5.9 & $40.1: 54.6: 0.6: 4.7$ & $7.7 \times 10^{18}$ & 1250 \\
S2 & 62 & 295 & 13.7 & $41.1: 52.0: 1.7: 5.2$ & $2.2 \times 10^{19}$ & 762 \\
S3 & 36 & 410 & 6.36 & $41.5: 51.0: 2.7: 4.8$ & $7.4 \times 10^{18}$ & 590 \\
\hline
\end{tabular}

\section{RESULTS AND DISCUSSION}

Temperature-dependent resistivity

Temperature-dependent resistivity is determined from room temperature down to $2 \mathrm{~K}$ and the results for three $\mathrm{Bi}_{2} \mathrm{Se}_{3}$ nanoribbons (S1, S2, and S3) are shown in Fig. 1a. The thickness, width, suspended length, and atomic ratios of all three nanoribbons are listed in Table 1 . The resistivity of all three nanoribbons exhibits a metallic behavior above $20 \mathrm{~K}$, indicated by $d \rho / d T \geq 0$. However, these three samples show distinct temperature dependence in the low temperature range, suggesting a disorderrelated phenomenon. The resistivity curve of S3 has a slight upturn below $20 \mathrm{~K}$ as shown in Fig. 1d. By contrast, the resistivity of S2 (Fig. 1c) decreases slightly when decreasing temperature from $20 \mathrm{~K}$ to $2 \mathrm{~K}$; and the resistivity of S1 (Fig. $1 \mathrm{~b}$ ) is nearly constant below $15 \mathrm{~K}$. S3 has the highest resistivity among three samples, followed by S1 and then S2. Notably, the increase in the resistivity of $\mathrm{S} 3$ below $20 \mathrm{~K}$ is owing to a $2 \mathrm{D}$ electron-electron interaction (EEI) effect in the weakly disordered regime. ${ }^{21}$ For S1 and $\mathrm{S} 2$, the signal of the 2D EEl effect becomes less discriminable because the bulk transport is dominant due to the relatively large thickness or the relatively high bulk carrier density in these two samples.
For the transport study of $\mathrm{Bi}_{2} \mathrm{Se}_{3} \mathrm{TI}$ nanoribbons, it is crucial to distinguish the surface state transport and the bulk state transport. One common approach is to conduct magnetic fieldorientation-dependent measurements because the quantum diffusion transport of TSSs responds strongly to a perpendicular magnetic field. In this work, we performed angle-dependent magnetotransport measurements on three nanoribbons, S1-S3, at temperatures from $2 \mathrm{~K}$ to $300 \mathrm{~K}$. In our measurements, $\theta=0^{\circ}$ is defined as a magnetic field perpendicular to both the sample surface and the excitation current, whereas $\theta=90^{\circ}$ denotes a magnetic field parallel to the excitation current, as illustrated in the inset of Fig. 2a. The angle-dependent magnetotransport properties of S1-S3 were first investigated under a magnetic field from $-9 \mathrm{~T}$ to $9 \mathrm{~T}$ at $2 \mathrm{~K}$ as shown in Fig. 2. Our experimental results show that the MR curves of all three samples dip sharply as the magnetic field approaches zero with specific quantum oscillations over the investigated magnetic field range. The quantum oscillation backgrounds show anomalous anisotropic MRs. The perpendicular-field MR has nonsaturating linear behavior for all samples, whereas the parallel-field MR demonstrates various behaviors in different samples. The results are systematically discussed below.

\section{Weak antilocalization}

A sharp dip in the MR curve is a characteristic feature of the WAL. The WAL effect, with a positive quantum correction to resistance, usually emerges in Tls in a low-strength magnetic field and originates from two factors. First, owing to the spin-momentumlocked Dirac cone that results from the strong spin-orbit coupling, surface states of a $\mathrm{TI}$ can acquire a $\pi$ Berry phase, which suppresses backscattering owing to the destructive interference between two closed paths with TRS. ${ }^{8}$ When a weak magnetic field is applied to break the destructive interference, a positive MR appears with a sharp dip, a key signature of 2D WAL. Second, the WAL can also occur in a strong spin-orbit coupling system, 

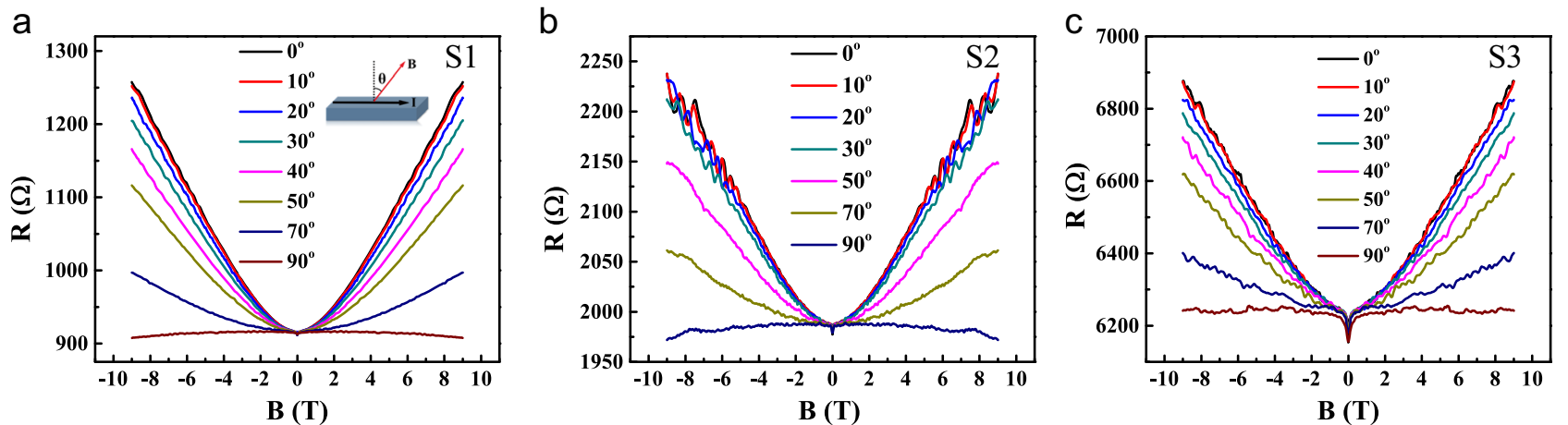

Fig. 2 MRs at $2 \mathrm{~K}$ for the magnetic field from $-9 \mathrm{~T}$ to $9 \mathrm{~T}$ and the tilt angle $\theta$ from $0^{\circ}$ to $90^{\circ}$. a S1. b S2. c S3. The inset of a shows the orientation of the magnetic field with $\theta=0^{\circ}$ for the perpendicular field and $\theta=90^{\circ}$ for the parallel field

a

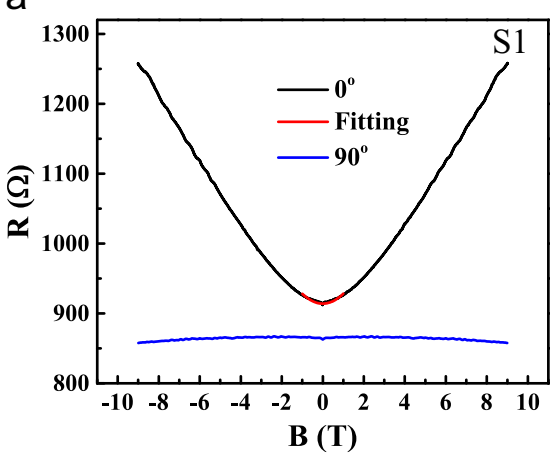

C

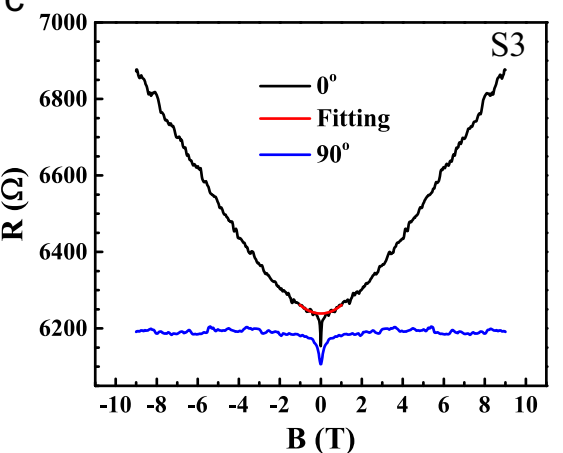

b

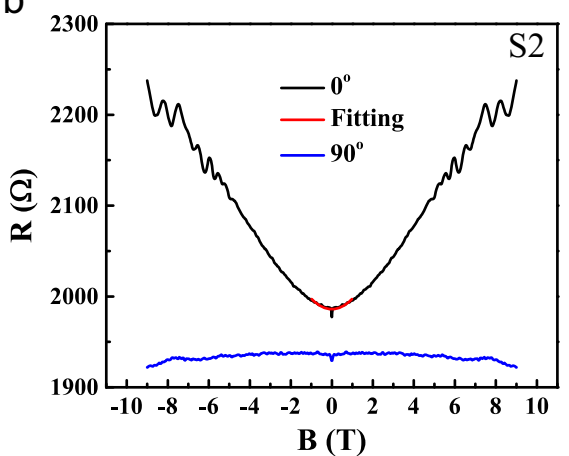

d

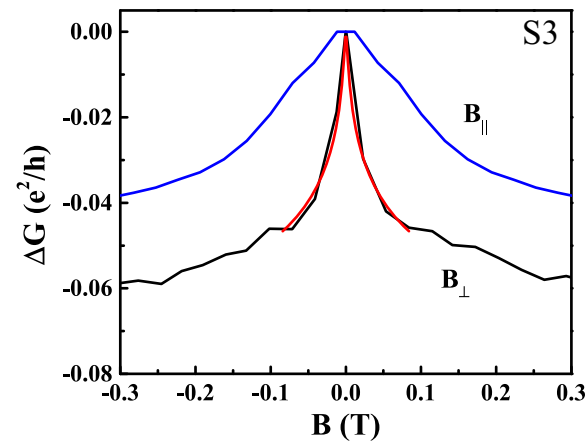

Fig. 3 a-c MRs in perpendicular (black) and parallel (blue) magnetic fields at $2 \mathrm{~K}$ for S1, S2, and S3. The parallel-field MR curves are offset by $-50 \Omega$ for the purpose of clarity. The red lines in a-c are the fitting curves of the perpendicular-field MR using Eq. (3) in the low magnetic field. d Variation of the magnetoconductance, $\Delta \mathrm{G}$, of $\mathrm{S} 3$ extracted from $\mathrm{c}$ using Eq. (2) for both perpendicular (black) and parallel (blue) fields. The red line is the fitting curve for the perpendicular field using Eq. (1)

including bulk states of Tls, where electrons acquire an additional phase $\pi$ by impurity scattering. ${ }^{22}$ If the Fermi energy lies in a bulk band induced by bulk doping, a WAL effect is still observed in the topologically nontrivial $2 \mathrm{D}$ electron system as long as the spinorbit coupling is of sufficient strength. A clear-cut separation of contributions from 2D surface states and bulk channels to the WAL is therefore challenging. The difference lies that 2D WAL can only be observed by applying a magnetic field with a component perpendicular to TSSs, whereas only bulk states can contribute to the quantum-interference correction in the parallel magnetic field orientation $\left(\theta=90^{\circ}\right)$.

The WAL is observed in all three samples (Fig. $3 a-c)$ even when the magnetic field is in the parallel orientation $\left(\theta=90^{\circ}\right)$, indicating the existence of 3D WAL from the bulk conductance. In S3, the WAL effect depends on the tilt angle $\theta$. It is most pronounced at $\theta=0^{\circ}$ and then becomes weaker as $\theta$ increases. The angle- dependent WAL effect and the substantial difference of WALs for perpendicular and parallel fields suggest the coexistence of 2D WAL from TSSs and 3D WAL from bulk transport. Although 3D WAL should be observed independent of the field orientation, to extract 2D WAL solely due to TSSs by subtracting 3D WAL in the parallel field from the WAL in the perpendicular field ${ }^{8}$ is questionable because the strong coupling between surface and bulk transport invalidates the simple subtraction.

On the other hand, the Zeeman effect ${ }^{23}$ and the EEl effect ${ }^{21}$ are known to make uneven contributions to the MR in perpendicular and parallel fields. The Zeeman and EEI effects are suppressed in the perpendicular-field MR owing to the ultra-short out-of-plane spin-orbit relaxation time and the mixing of up and down spin channels quenching the spin-triplet term, respectively, both of which are caused by the strong spin-orbit coupling. Hence, the change in the perpendicular-field MR can be described using the 
Hikami-Larkin-Nagaoka (HLN) theory under the low-field limit. ${ }^{24}$ However, the contributions of the Zeeman and EEl effects on the parallel-field MR could be substantial for relatively long in-plane spin-orbit relaxation times. In addition, both the Zeeman and EEI effects produce a parallel-field MR that has parabolic dependence for the low-strength field and logarithmic dependence for the high-strength field, which is clearly not the case for S1-S3. Therefore, the dip in the parallel-field MR is only owing to the WAL from bulk transport rather than the Zeeman and EEI effects.

As the Zeeman and EEI effects can be neglected, the HLN equation $^{25}$

$\Delta \mathrm{G}=\frac{W}{L}\left[-\frac{a e^{2}}{2 \pi^{2} \hbar}\right]\left[\ln \left(\frac{\hbar}{4 e L_{\varphi}^{2} \mathrm{~B}}\right)-\psi\left(\frac{1}{2}+\frac{\hbar}{4 e L_{\varphi}^{2} \mathrm{~B}}\right)\right]$,

is used to approximately fit the curve of the variation of magnetoconductance $(\Delta G)$ for the perpendicular field in the low-strength field where the WAL occurs. $\Delta \mathrm{G}$ can be determined by

$\Delta \mathrm{G}=\frac{1}{\mathrm{R}(\theta, \mathrm{B})}-\frac{1}{\mathrm{R}(\theta, 0)}$,

where $R(\theta, B)$ is the $M R$ at the field strength $B ; R(\theta, 0)$ is the MR when $\mathrm{B}=0$. The obtained $\Delta \mathrm{G}$ curves in the perpendicular (black) and parallel (blue) fields are illustrated in Fig. 3d for S3. In Eq. (1), $\Psi$ is the digamma function; $L_{\varphi}$ is the phase coherence length; and $a$ is a prefactor that is related to the nature of electrons. In a system with a single conduction channel, $a$ is universal and equals -0.5 . In a system with two independent parallel conduction channels, $a$ $=-1.0$. The HLN fitting was performed for the perpendicular-field $\Delta \mathrm{G}$ curve as shown in Fig. $3 \mathrm{~d}$ (red line). The fitting parameters, $a$ and $L_{\varphi}$, are determined to be -0.6785 and $623 \mathrm{~nm}$, respectively. If two surface and one bulk conduction channels are fully decoupled, a should be equal to -1.5 . However, the extracted value of $a$ is close to -0.5 , suggesting that electrons on the surface can be coupled to bulk states and the entire film effectively acts as one channel contributing to the 2D WAL effect. This is confirmed by the extracted $L_{\varphi}(623 \mathrm{~nm})$, which is considerably larger than the thickness of S3 ( $36 \mathrm{~nm})$, indicating the existence of strong coupling between surface and bulk transport. The dips do exist in the MR curves of S1 and S2 but are subtle for all the field orientations, which manifests the contribution from bulk transport. However, the contribution from TSSs cannot be confirmed for S1 and S2.

Notably, if ignoring the dip near $B=0$, the $M R$ has an approximate $B^{2}$ dependence in the low magnetic field range $(|\mathrm{B}|<1 \mathrm{~T})$. This may tentatively be ascribed to the classical MR and approximated by ${ }^{26}$

$$
[R(B)-R(0)] / R(0)=(\mu \cdot B)^{2} \text {. }
$$

Thus, through the curve fitting, we obtained the mobility $\mu$ as 1250,762 , and $590 \mathrm{~cm}^{2} / \mathrm{V} \cdot \mathrm{S}$ for S1, S2, and S3, respectively. The electron concentration $n=1 /(\rho|e| \mu)$ is then calculated to be $7.7 \times$ $10^{18}, 2.2 \times 10^{19}$, and $7.4 \times 10^{18} \mathrm{~cm}^{-3}$ for S1, S2, and S3.

It is important to point out that, although the 2D WAL in S3 indicates the surface conduction, it does not necessarily arise (only) from TSSs. As previously reported, slow migration of Se vacancies close to the surface leads to the increased concentration of positively charged impurities, which induce time-dependent bulk band bending in $\mathrm{Bi}_{2} \mathrm{Se}_{3}$ and create a potential well that results in 2DEG near the surface, as confirmed by angle-resolved photoemission spectroscopy. ${ }^{20}$ It is impossible to clearly separate TSSs and 2DEG only from WAL observations. To facilitate the discussion and analysis, if 2DEG is treated to be separated from bulk states and TSSs, the 2D WAL is the collective effects of transport of TSSs and 2DEG.

\section{Shubnikov-de Haas oscillations}

SdHOs are clearly observed in S1 and S2, as shown in Fig. 2a, b. To analyze SdHOs in S1, we subtract the MR background from the raw $R(B)$ data and plot the residual oscillatory part of the $M R$ as a function of $B_{\perp}^{-1}=[B \cos \theta]^{-1}$ in Fig. $4 a$, where $\theta$ is the angle between the magnetic field and the unit vector normal to the current direction. Several equidistant maxima (indicated by dark blue dash lines) are easily recognized, and crucially, the positions of the maxima depend only on the field component in the perpendicular direction $B_{\perp}$, which signifies that the observed oscillations are $2 \mathrm{D}$ nature. In addition, the oscillations are not resolved for $\theta>40^{\circ}$. However, for bulk transport, SdHOs are expected for all orientations of the magnetic field. By comparing the magnetic field values at the SdHO peaks, we can plot the Landau index $\mathrm{N}$ of the $\mathrm{SdHO}$ peak positions versus $\mathrm{B}_{\perp}^{-1}$ and $\mathrm{a}$ linear dependence is obtained as shown in Fig. 4b. Although both 2DEG in conventional semiconductors and 2D Dirac electrons have linear $N$ versus $B_{\perp}^{-1}$ dependence, their difference in the Berry's phase renders an intercept of 0 for a regular 2DEG and -0.5 for Dirac electrons with non-zero Berry phase. ${ }^{23}$ The linear fitting curve of $\mathrm{N}\left(\mathrm{B}_{\perp}^{-1}\right)$ in Fig. $4 \mathrm{~b}$ has an N-intercept of $-0.008 \pm$ 0.01 , indicating that SdHOs unambiguously originate from 2DEG. An additional oscillation peak (indicated by a brown dot line) has been clearly differentiated from those attributed to 2DEG at the high magnetic field. The Fermi level should be located close to the bulk band edge for S1 with a carrier density $\sim 10^{18} \mathrm{~cm}^{-3}$ and the observed SdHOs could be the superposition of signals from electrons in TSSs, 2DEG, and bulk states. However, as the period of $\mathrm{SdHOs}$ depends inversely on the cross sectional area of the Fermi surface, it will be difficult to observe SdHOs from a relatively large Fermi surface. $^{23,27,28}$ Therefore, the result confirms that 2DEG has the dominant role in the collective SdHO signals, which may be because of the relatively small cross sectional area of its Fermi surface and the relatively high carrier density and mobility. The general expression for SdHOs can be described by ${ }^{23}$

$$
\Delta \mathrm{R}(\mathrm{B})=A \exp \left(-\frac{\pi}{\mu \mathrm{B}}\right) \cos 2 \pi\left(\frac{\mathrm{B}_{\mathrm{F}}}{\mathrm{B}}-\frac{1}{2}+\beta\right),
$$

where $B_{F}$ is the frequency of SdHOs in $1 / B$, and $\beta \times 2 \pi$ is the Berry's phase. According to Eq. (4), the amplitude of SdHOs grows exponentially with the magnetic field. Considering that the carrier motility $\mu$ of TSSs is just slightly higher than that of 2DEG, the amplitudes of SdHO signals from TSSs and 2DEG may not be so distinct at the low magnetic field. However, as the magnetic field becomes relatively high, the oscillation peak from TSSs can be differentiated from those of $2 \mathrm{DEG}{ }^{29}$ Therefore, the additional oscillation peak can be ascribed to TSSs.

By contrast, the pronounced oscillations in S2 are complex and have two sources, as illustrated in Figs. $2 \mathrm{~b}, 4 \mathrm{c}$. When $|\mathrm{B}|>5 \mathrm{~T}$, the amplitude of SdHOs is prominent and the oscillations can be easily distinguished for any given field orientation, suggesting a 3D origin. When $\theta$ goes to $90^{\circ}$, the amplitude of SdHOs decreases significantly. Further studies revealed that the positions of maxima and minima demonstrate title angle dependence, however, gradually deviate from the $1 / \cos \theta$ trend even when $\theta \leq 30^{\circ}$, as shown in Fig. $4 \mathrm{~d}$. The oscillations for $\theta$ in the range of $50^{\circ}-90^{\circ}$, compared with those of small angles, have relatively large magnetic field frequency and become much more gentle with $\Delta \mathrm{R}$ saturating at a value which is $80 \%$ less than that at $\theta=0^{\circ}$. This is owing to the reason that the period of the Fermi surface of bulk states usually increases when the magnetic field changes from the perpendicular direction to the parallel direction. ${ }^{7}$ However, our observation contradicts the results reported by Cao et al. ${ }^{29}$ for heavily doped metallic $\mathrm{Bi}_{2} \mathrm{Se}_{3}$, in which individual quintuple layers in the bulk act as parallel 2D electron transport channels and thus 2D SdHOs are observed. 
a

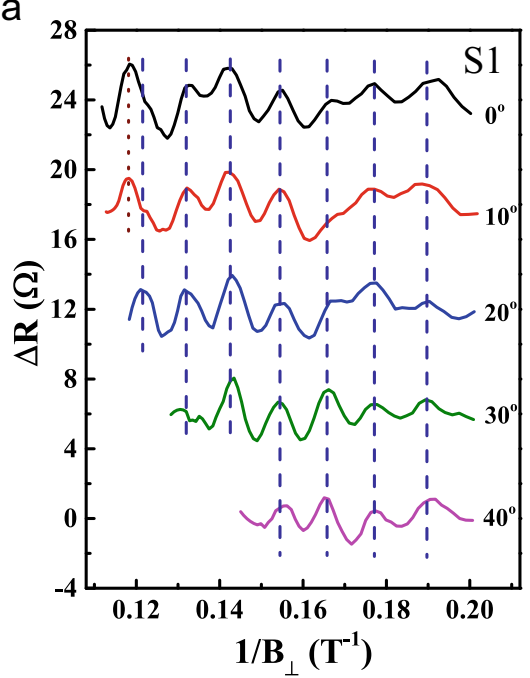

C

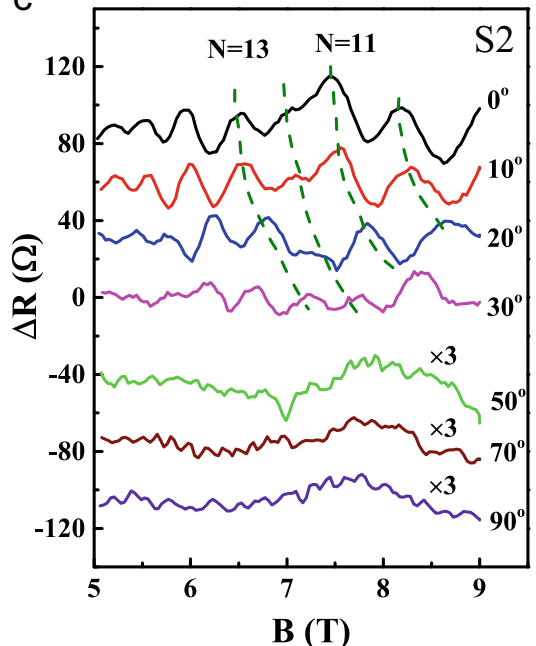

b

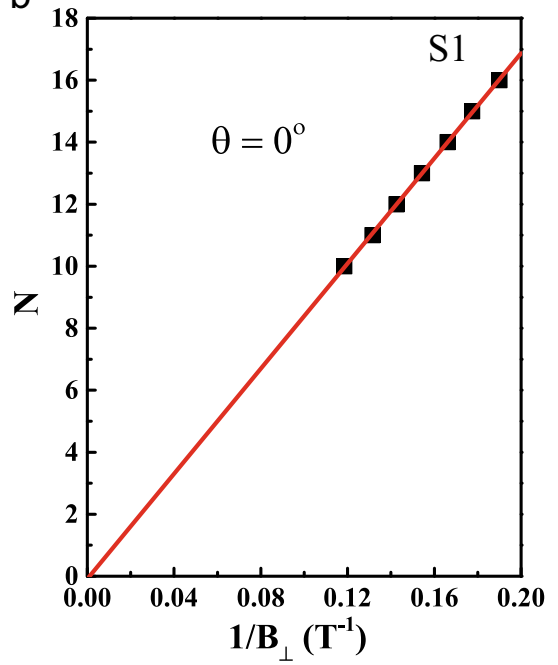

d

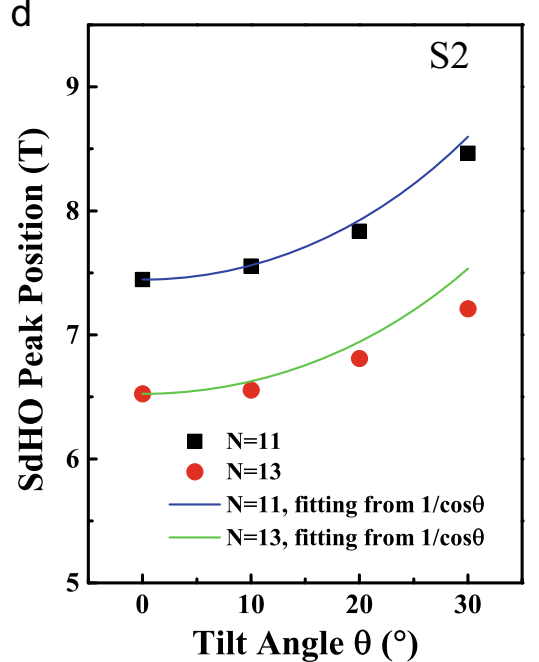

Fig. 4 a SdHOs after subtracting the background MR in $\mathrm{S} 1$ for $\theta$ ranging from $0^{\circ}$ to $40^{\circ}$. The data are consistent with the $1 / \cos \theta$ dependence, which is expected for 2D SdHOs. The dark blue dash lines point to oscillation peaks from 2DEG, whereas the brown dot line points to the oscillation peak from TSSs. b Landau index N plotted against the inverse of the perpendicular component of the magnetic field for SdHOs in the perpendicular field $\left(\theta=0^{\circ}\right)$ in S1. The intercept of the linear fitting curve is -0.008 , indicating a 2DEG characteristic. c SdHOs after subtracting the background MR in S2 for $\theta$ ranging from $0^{\circ}$ to $90^{\circ}$, which demonstrate a 3D Fermi characteristic. For the purpose of clarity, $\mathrm{SdHOs}$ for $50^{\circ}, 70^{\circ}$, and $90^{\circ}$ are amplified by three times. d Positions of SdHO peaks with $\mathrm{N}=11$ or 13 plotted against the tilt angle $\theta$, which slightly deviate from the $1 / \cos \theta$ dependence and rule out their $2 \mathrm{D}$ characteristic

\section{Universal conductance fluctuation}

A series of tiny peaks and dips superimposed on SdHOs can be observed for $\theta=50^{\circ}, 70^{\circ}$, and $90^{\circ}$ in $\mathrm{S} 2$ (Fig. $2 \mathrm{~b}, 4 \mathrm{c}$ ). In fact, these magnetofingerprints can also be observed in $\mathrm{S} 1$ when the magnitude field is nearly parallel to the current direction as shown in Fig. 2a. The details presented in Fig. $2 \mathrm{~b}$ illustrate that the amplitude of these tiny oscillations becomes obvious when turning the magnetic field to the orientation parallel to the current direction, but gradually fades as $\theta$ goes to $0^{\circ}$. It seems that the tiny resistance oscillations demonstrate a dependence on $\mathrm{B}_{\|}=\mathrm{B} \sin \theta$, which is usually a characteristic of the AharonovBohm (AB) or Altshuler-Aronov-Spivak (AAS) effect. However, similar magnetofingerprints can also be observed in $\mathrm{S} 3$ in any field direction with similar amplitudes (Fig. 2c and Fig. 5a), which cannot be simply ascribed to $\mathrm{SdHO}$ s because of their intensive shock starting from extremely low magnetic field $(|B|<1 \mathrm{~T})$. We are inclined to ascribe these features to UCFs for the following reasons. First, these magnetofingerprints are retraceable and aperiodic fluctuations versus $B$, distinguishable from random noise. Similar features are observed in MR curves of all three samples measured at different temperatures, indicated by some specific "fingerprints" in Fig. 2a-c and Fig. 6. The AB or AAS effect can be excluded because they are periodic in $h / e$ or $h / 2 e$, respectively. Second, the root-mean-square (rms) amplitude $\delta G_{\text {rms }}=\sqrt{\left\langle\left[\delta G(B, T)-\left\langle\delta G_{0}(B, T)\right\rangle\right]^{2}\right\rangle}$, where $\langle\cdots\rangle$ represents the ensemble average, is nearly unchanged between $1 \mathrm{~T}$ and $9 \mathrm{~T}$ with a unit of $\mathrm{e}^{2} / \mathrm{h}$ (Fig. 5a), however, the amplitudes of SdHOs grow exponentially. The amplitudes of the UCF signals are dependent upon the size of the sample. Because S3 has the smallest thickness among three samples, its UCF signals may have been sufficiently strong to mix with the possible SdHO signals as demonstrated in Fig. 2c and Fig. 5a.

UCFs are thought to imply the field modulation caused by the interference of variations of disordered configurations in the conduction channels. In Tls, the transport channel is determined by the position of the Fermi level. Specifically, if the Fermi level is in the band gap, only surface channels contribute to the transport 

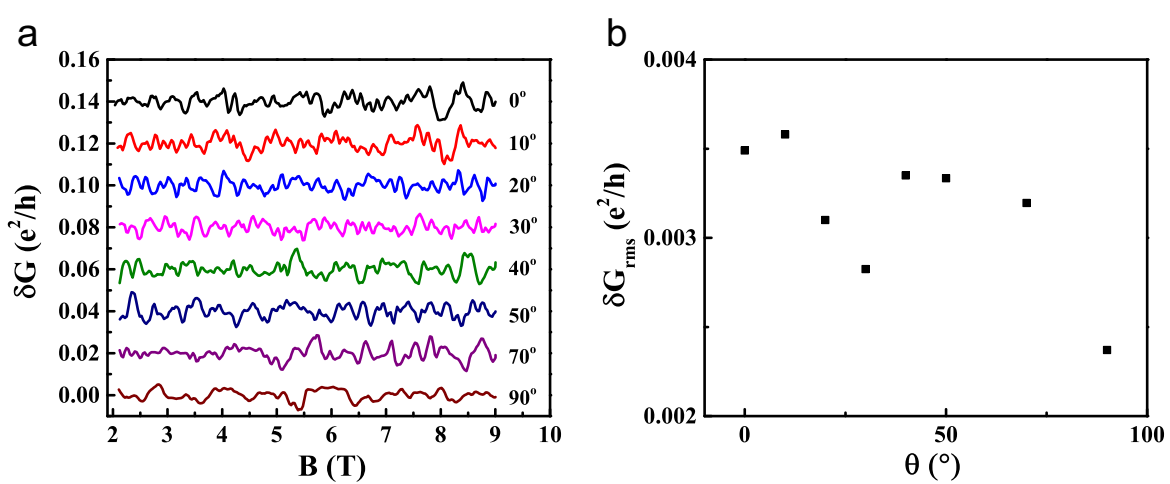

Fig. 5 Conductance fluctuations of S3. a Conductance fluctuations at $2 \mathrm{~K}$ for $\theta$ from $0^{\circ}$ to $90^{\circ}$. $\mathbf{b}$ Dependence of $\delta G_{\mathrm{rms}}$ on the tilt angle $\theta$
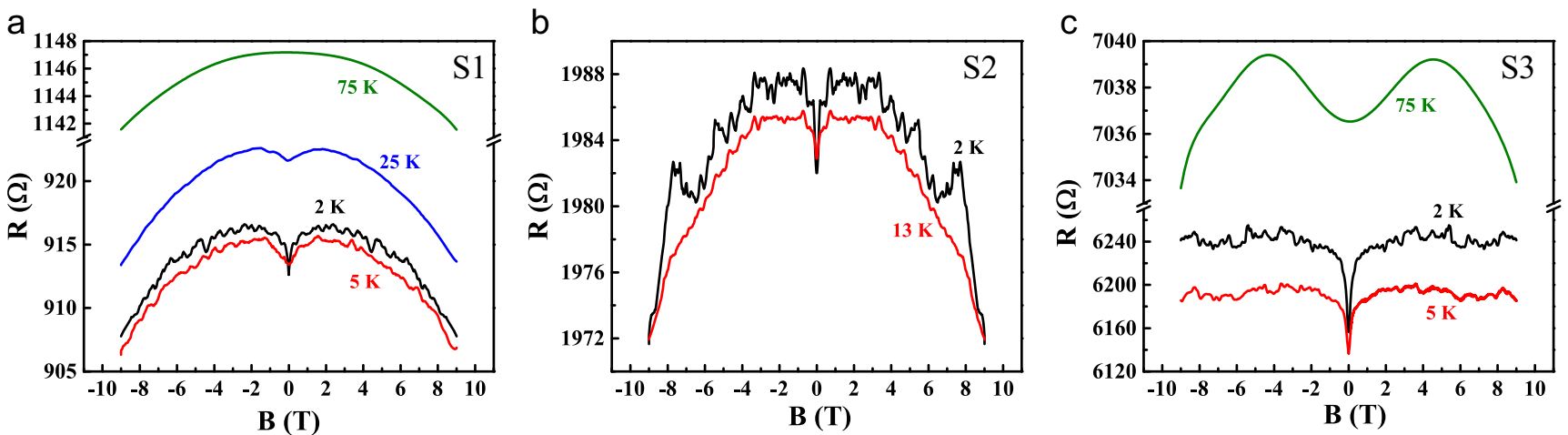

Fig. 6 a MRs of S1 in the parallel magnetic field from $2 \mathrm{~K}$ to $75 \mathrm{~K}$. b MRs of S2 in the parallel magnetic field at $2 \mathrm{~K}$ and $13 \mathrm{~K}$. c MRs of S3 in the parallel magnetic field from $2 \mathrm{~K}$ to $75 \mathrm{~K}$

and UCFs have 2D nature. Conversely, if the Fermi level is at the bottom of the conduction band, then the bulk, 2DEG, and TSSs will contribute to the transport with both $3 \mathrm{D}$ and $2 \mathrm{D}$ characteristics. Our samples have metallic characteristics, so UCFs should mainly come from the bulk and 2DEG. However, abnormal phenomena still exist, which require the further study. The amplitude of UCFs in S3 does not strictly demonstrate the $B_{\perp}=B \cos \theta$ dependence maybe because they are mixed with other oscillation signals such as SdHOs. The UCFs in S1 and S2 are more prominent in the parallel field and gradually disappear as close to the perpendicular field, whereas UCFs in S3 are obvious in all directions with the amplitude increasing as close to the perpendicular field (Fig. 5b). One possible reason is that, as S3 is thinner than S1 and S2, UCFs in the bulk and 2DEG are both significant in S3 and thus have superimposed characteristics of 2D and 3D transport. However, UCFs from the bulk dominate in S1 and S2. UCFs become stronger in the parallel-field MR because the perpendicular-field MR reflects surface transport. Further study is still needed to understand UCFs in Tls.

\section{Background magnetoresistances}

In addition to quantum transport phenomena such as WAL, SdHOs, and UCFs, strong background MRs are also observed in $\mathrm{TI}$ nanoribbons, which exhibit anisotropy with respect to the magnetic field direction (Fig. 2a-c). The perpendicular-field MR exhibits nonsaturating positive and linear behavior that persists to room temperature (Fig. 2a-c). In the past few years, a lot of research has focused on unraveling the mechanism behind this LMR and investigating whether TSSs are the origin of the LMR and which model can be applied. However, the nature of the LMR in Tls has not been determined yet. The positive evidences supporting that TSSs are crucial for the LMR are revealed by transport measurements including the observation of 2D SdHOs overlapping with the $L M R,{ }^{7,10}$ the significant decrease of the LMR by changing from the perpendicular field to the parallel field with a $\cos \theta$ dependence, ${ }^{30}$ enhanced LMR by tuning the Fermi level into the bulk band gap, ${ }^{31}$ and the introduction of a gap in the Dirac cone to quench the LMR in ultrathin TI films. ${ }^{32}$

On the premise of ascribing the LMR to TSSs, attempts have been made to explain the observation of the LMR by the quantum LMR model of Abrikosov. ${ }^{11,32}$ However, the carrier density in TIs is relatively large and, for realistic magnetic field values, more than one Landau levels are usually occupied, indicating that the system is far away from the extreme quantum limit with only one Landau magnetic band filled to which Abrikosov's model can be applied. ${ }^{33}$ To explain the LMR observed in $\mathrm{Bi}_{2} \mathrm{Se}_{3}$ nanosheets, ${ }^{10}$ Wang and $\mathrm{Lei}^{34}$ derived a different model using the balance-equation approach and pointed out that the LMR could also arise in 2D systems with a linear energy dispersion and a non-zero $g$ factor in the presence of overlapping Landau levels. Although it is tempting to attribute the LMR to TSSs and apply the quantum LMR model, there exist some difficulties, which cannot be overcome. First, by analyzing the resistivity measurement, the Hall measurement, and $\mathrm{SdHOs}$ for our samples and those in the literature, ${ }^{10,30-32}$ it can be found that the bulk conductance is dominant compared with the surface conductance. Therefore, when a large $2 \mathrm{D}$ effect is seen on the scale of the total conductance, it seems unlikely that the LMR only originates from surface states. Gao et al. investigated the MR as a function of the electrical gate voltage in $\mathrm{Bi}_{2} \mathrm{Se}_{3}$ sheets ${ }^{31}$ and concluded that the MR was the combined effects of the gap and bulk state resistance values. Second, 2D SdHOs with an intercept of 0 or -0.5 , can also be observed in highly doped $\mathrm{Bi}_{2} \mathrm{Se}_{3}$ samples $^{30}$ as well as our samples. Thus, solely observing the $|\cos \theta|$ dependence is not sufficient to attribute the magnetotransport effect to surface states. More stringently, it just indicates a 2D origin. Third, even the model developed by Wang and $\mathrm{Lei}^{34}$ 
assumes that the bulk contribution is negligible, which is far away from the real case.

On the other hand, some researchers ${ }^{31,35}$ claim that the LMR in Tls can be explained by the Parish-Littlewood model, the classical inhomogeneity model, which suggests that the inhomogeneity in disordered conductors can give rise to the LMR as shown in $\beta-\mathrm{Ag}_{2} \mathrm{Te}^{36,37}$ However, as most of TI samples have high crystalline quality, it is unlikely that the observed LMR can be explained by the Parish-Littlewood model. ${ }^{10}$ Even for those samples containing a lot of defects, the conventional Parish-Littlewood model has still been excluded to explain the LMR because there is a disagreement between the range of the mobility and the range of the LMR. $^{38}$ However, it should be emphasized that although structural and compositional inhomogeneity can be neglected, the electronic inhomogeneity in Tls is obvious and significant. At least three conduction channels related to TSSs, 2DEG from band bending, and bulk states contribute to electron transport even if neglecting the contribution from impurity bands. The electronic inhomogeneity may be responsible for the LMR, ${ }^{39,40}$ however, more effort is needed to modify the Parish-Littlewood model and to investigate under which conditions it can be applied to Tls.

The parallel-field MRs of three samples, however, show markedly distinct properties. Negative MRs have been observed for S1 and S2, as shown in Figs. 6a, b, respectively, which roughly have a $B^{2}$ dependence. Negative MR phenomena are rare in nonmagnetic material systems and are mainly observed in various disordered quantum systems in both weak- and stronglocalization regimes. ${ }^{41,42}$ However, such quantum interference generally appears under cryogenic conditions, because there is a reduction in the phase coherence at higher temperatures owing to enhanced inelastic electron-phonon and electron-electron scatterings. Accordingly, the quantum transport behavior of weak localization may be weakened and eventually disappear when temperature is increased, as it occurs for WAL. Our experimental results demonstrate that as the temperature increases, the lowfield WAL cusp becomes less pronounced, indicating the progressive suppression of the quantum correction effect in the bulk conduction channel. However, the negative parallel-field MR is still observable at high temperatures and at high magnetic fields, as illustrated in Fig. 6a. Notably, the parallel-field MR of S3 exhibits the WAL in the low-strength field, but becomes positive in the mid- and high-strength fields at $2 \mathrm{~K}$ (Fig. $6 \mathrm{c}$ ); whereas the MR is positive in the low-strength field and turns to negative in the highstrength field when the temperature increases.

Various behaviors of the parallel-field MR in the $\mathrm{Tl} \mathrm{Bi}_{2} \mathrm{Se}_{3}$ have been observed, but have not been systematically studied. The negative $M R$ in the parallel magnetic field was also observed in pure $\mathrm{Bi}_{2} \mathrm{Se}_{3}$ nanoribbons with a thickness of $100 \mathrm{~nm}^{10}$ and in a thin film with a thickness of $150 \mathrm{~nm}^{43}$ from $2 \mathrm{~K}$ to $300 \mathrm{~K}$. The $A B$ effect with the positive MR at $2 \mathrm{~K}$ was reported for $\mathrm{Bi}_{2} \mathrm{Se}_{3}$ nanoribbons with a thickness of $30-50 \mathrm{~nm},{ }^{17}$ which is also demonstrated in our sample S3. Wang et al. ${ }^{44^{\prime}}$ reported that the positive MR was observed in the low-strength field but it switched to the negative MR in the sufficiently high-strength field in $\mathrm{Bi}_{2} \mathrm{Se}_{3}$ thin films with a thickness of 45-200 QL. Combining these results with the phenomena observed in our samples, it is clear that the parallel-field $\mathrm{MR}$ in $\mathrm{Bi}_{2} \mathrm{Se}_{3}$ could be negative or positive, or even tuned from positive to negative with the increase of the magnetic field strength. Wang et al. ${ }^{43}$ suggested that $\mathrm{Bi}_{\text {se }}$ antisite defects induced local magnetic moments that were realigned when a magnetic field was applied, reducing the spin-dependent scattering and resulting in a decrease in the total resistance of the $\mathrm{Bi}_{2} \mathrm{Se}_{3}$ sample. However, this simple argument concerning defects could not account for various behaviors of the parallel-field MR in this study. It also contradicts the fact that other Bi-chalcogenide Tls, such as $\mathrm{Bi}_{2} \mathrm{Te}_{3}$, possess antisite defects ${ }^{45}$ but only exhibit the positive MR in a parallel magnetic field. ${ }^{24}$
The unique but complex behaviors of the parallel-field MR in $\mathrm{Bi}_{2} \mathrm{Se}_{3}$ indicate that competing and compensating factors may affect the MR. It is reasonable to guess that the parallel-field MR is the collective results of a positive MR and a negative MR existing in different conductance channels. A thorough understanding of the results presented in this study may require the treatment of the collective effects of surface and bulk states.

Transport measurements have revealed that the collective spin polarization of TI surface states is aligned by the current. ${ }^{43}$ When the magnetic field is perpendicular to the current, it is parallel to the spin polarization direction; conversely, when the field is parallel to the current, it is perpendicular to the spin polarization of the surface current. In an ideal 3D TI thin film in which each of top and bottom surfaces can be treated as a 2D system, the principal effect of a parallel field is to simply shift surface states in momentum space. The Zeeman and EEl effects contribute to a positive MR at low temperature. ${ }^{24}$ At high temperature, the Lorentz force deflects surface electrons, leading to a positive MR that results from the classical galvanomagnetic effect. ${ }^{43}$

If TSSs contribute to a positive MR, the source of a negative MR in a parallel field is clearly either owing to 2DEG near TSSs or owing to bulk states. Recently, triggered by the discovery of Weyl semimetals $\mathrm{TaP}^{46}$ and $\mathrm{TaAs}^{47}$ and the Dirac semimetals $\mathrm{Na}_{3} \mathrm{Bi}^{48}$ and $\mathrm{Cd}_{3} \mathrm{As}_{2}$, it has been proposed that a quantum mechanical phenomenon called the axial anomaly can give rise to a negative MR. Remarkably, further calculations propose that the axial anomaly is a universal phenomenon for a generic 3D metal or for 3D electrons in a semiconductor in the presence of parallel electric and magnetic fields only if ionic impurities exist and the resulting impurity scattering contributes a strong negative $M R \propto$ $\mathrm{B}^{2}$ in the quantum limit. ${ }^{50,51}$ On the other hand, as mentioned previously, the negative parallel-field $\mathrm{MR} \mathrm{in} \mathrm{Bi}_{2} \mathrm{Se}_{3}$ is different from the common positive MRs in other Tls, whereas the unique characteristic of $\mathrm{Bi}_{2} \mathrm{Se}_{3}$ is that native Se-vacancy defects are kind of ionic impurities dominating bulk transport. We hypothesize that the negative $\mathrm{MR}$ in $\mathrm{Bi}_{2} \mathrm{Se}_{3}$ is owing to the scattering mechanism related to Se vacancies.

To determine the contribution of 2DEG to the parallel-field MR, the physical nature of 2DEG near TSSs should be revealed. TSSs are odd numbers of massless Dirac cones on the surface, and electrons in TSSs are spin-locked to momentum and protected from backscattering. The presence of 2DEG near TSSs opens decay channels for TSSs and permits the scattering between 2DEG and TSSs. The 2DEG should certainly be spin degenerate and could be prone to such instabilities. Previous studies have also indicated that hybridization between TSSs and 2DEG may exist and that 2D bulk gap states will partly display a large Rashba coupling that locks the e spin transverse to . $^{12}$ Because electrons in the potential well caused by band bending share unusual properties of those in TSSs, it can be concluded that ionic scattering, if exists, should be partly quenched in the potential well near TSSs. However, it is too rushed to conclude that the parallel-field MR in 2DEG is positive. The potential well from the surface band bending is usually nonsymmetrical, and there exists a potential gradient from the surface to the inner-part of the bulk. For 2DEG close to the bulk, the hybridization between TSSs and 2DEG should become less significant if the thickness of the potential well is large enough. Recently, a direct measurement of the band bending on $\mathrm{Bi}_{2} \mathrm{Se}_{3}$ by using the bulk sensitive angular-resolved hard X-ray photospectroscopy reveals that the band bending extends up to $\sim 20 \mathrm{~nm}$ into the bulk, indicating that only samples with a thickness larger than $40 \mathrm{~nm}$ can demonstrate bulk transport ${ }^{52}$ although the critical thickness can be tuned by doping or environment. Interestingly, as reported in previous reports and our experiments, films or nanoribbons with a thickness larger than $60 \mathrm{~nm}$ usually demonstrate negative MRs, however, nanoribbons with a thickness smaller than $50 \mathrm{~nm}$ usually demonstrate positive MRs. Therefore, we propose that the negative MR is presumably 


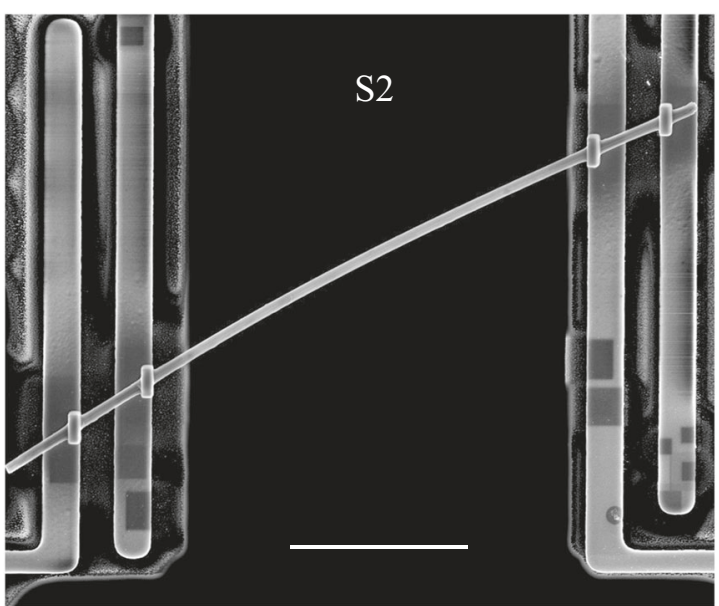

Fig. 7 SEM image of a $\mathrm{Bi}_{2} \mathrm{Se}_{3}$ nanoribbon (S2) on a suspended micro-device for the MR measurement. The scale bar is $5 \mu \mathrm{m}$

owing to ionic scattering in the bulk channel. When the negative MR "overcomes" the positive MR effect from the TSSs channel, the collective effects result in the negative MR behavior in thick samples. Otherwise, the MR in the parallel field should be positive just decided by TSSs and 2DEG in thin samples. 2DEG is in the transition region from TSSs to the bulk with the parallel-field MR balanced from the positive value owing to the hybridization effect on the TSSs-2DEG interface side and the negative value on the 2DEG-bulk interface side. When the temperature is increased, bulk carriers are activated. The negative resistance from the bulk will gradually dominate. And the collective MR in the parallel field will gradually change from positive to negative as shown in Fig. $6 \mathrm{c}$ in S3.

In conclusion, this work is focused on transport characteristics related to TSSs, 2DEG near the surface, and bulk states in Sb-Tecodoped $\mathrm{Bi}_{2} \mathrm{Se}_{3} \mathrm{TI}$ nanoribbons with different carrier densities and surface-area-to-volume ratios. A series of quantum signals, including WAL, SdHOs, UCFs, and anisotropic MRs, have been observed and extensively discussed for their origins. Three measured samples can be ranked as S2, S1, and S3 according to the descending order of the carrier density. S2 is heavily doped with a carrier density about three times of those of S3 and S1. Thus, the Fermi level should be deeply pinned in the bulk band in S2. In contrast, Fermi levels in S1 and S3 will be more close to the band edge. On the other hand, S3 has the highest surface-area-tovolume ratio, which is $\sim 1.5 \times$ and $2 \times$ those of S2 and S1, respectively. Therefore, it is reasonable to observe that surface transport signals are more pronounced in S3 and S1, whereas bulk transport characteristics are the most distinct in S2. In S2, SdHOs have the bulk Fermi characteristics, which is different from the 2D layered transport reported previously. The parallel-field MR of nanoribbons can be tuned from positive to negative, which is attributed to the collective effects of a positive MR owing to TSSs transport and a negative MR presumably owing to the axial anomaly, resulting from long-range ionic impurity-scattering processes in bulk carriers.

\section{METHODS}

Materials synthesis

The Sb-Te-codoped $\mathrm{Bi}_{2} \mathrm{Se}_{3}$ single-crystal TI nanoribbons were synthesized in a Lindberg three-zone horizontal tube furnace through a vapor-liquid-solid method. $\mathrm{Bi}_{2} \mathrm{Se}_{3}$ and $\mathrm{Sb}_{2} \mathrm{Te}_{3}$ powders (99.999\%, Aldrich) were placed in the hot center of the tube furnace. A silicon wafer, coated with gold nanoparticles as catalysts, was used as the growth substrate and placed in the cold zone of the furnace, which is $\sim 15 \mathrm{~cm}$ away from the center. The tube was initially pumped down to a base pressure of $\sim 0.01$ mbar. With a flow of $25 \mathrm{sccm}$ argon gas, the hot center of the furnace was heated to $580^{\circ} \mathrm{C}$ and then held at this temperature for $\sim 120 \mathrm{~min}$ at a pressure of $250-300$ mbar. The temperature of the substrate was maintained at $\sim 450^{\circ} \mathrm{C}$ during the growth. Subsequently, the furnace was cooled down naturally. A layer of Sb-Te-codoped $\mathrm{Bi}_{2} \mathrm{Se}_{3}$ nanoribbons was formed on the substrate. Detailed information regarding the crystal orientation and EDX spectra is presented in Fig. S1 in Supplementary Information.

\section{Magnetotransport measurements}

A unique micro-device, which was originally used to study thermoelectric transport properties of nanowires, ${ }^{53}$ is adopted in this work to characterize the MR of individual $\mathrm{Bi}_{2} \mathrm{Se}_{3}$ nanoribbons. The scanning electron microscopy (SEM) image of a nanoribbon (S2) on a suspended device is shown in Fig. 7. The device consists of two side-by-side suspended membranes with a spacing of several microns. Two platinum electrodes are patterned on each membrane. An individual $\mathrm{Bi}_{2} \mathrm{Se}_{3}$ nanoribbon is placed to bridge two membranes by using a micromanipulator. The four platinum electrodes on two suspended membranes enable the four-probe measurement of the electrical resistance of the nanoribbon. To ensure good electrical contacts between the $\mathrm{Bi}_{2} \mathrm{Se}_{3}$ nanoribbon and all four platinum electrodes, platinum/ carbon composites are locally deposited on top of each contact using the electron-beam-induced deposition, as shown in Fig. 7. The design of the suspended micro-device enables us to determine transport properties with no effect from the substrate in a physical property measurement system.

\section{DATA AVAILABILITY}

The data that support the findings of this study are available from corresponding authors on reasonable request.

\section{ACKNOWLEDGEMENTS}

We acknowledge the financial support from the Research Grants Council of the Hong Kong Special Administrative Region, People's Republic of China, under the General Research Fund (RGC Ref No. 14238416), the National Natural Science Foundation of China (Grant No. 51676036), and the Starting Research Fund from the Wuhan University of Technology.

\section{AUTHOR CONTRIBUTIONS}

H.T. and J.J. synthesized the nanoribbons. H.T., Y.X., and K.D. prepared the samples for magnetoresistance measurements. X.W. fabricated the micro-devices. H.T. and X.Y. conducted magnetoresistance measurements. H.T., Y.X., and F.Q. performed the SEM and TEM characterization. Y.Z. carried out the EBID treatment. J.Y., M.L., and D.X. supervised the project. H.T. wrote the manuscript. H.T., Y.X., and D.X. edited the manuscript. All authors discussed the results and commented on the manuscript.

\section{ADDITIONAL INFORMATION}

Supplementary information accompanies the paper on the npj Quantum Materials website (https://doi.org/10.1038/s41535-018-0142-z).

Competing interests: The authors declare no competing interests.

Publisher's note: Springer Nature remains neutral with regard to jurisdictional claims in published maps and institutional affiliations.

\section{REFERENCES}

1. Zhang, $\mathrm{H}$. et al. Topological insulators in $\mathrm{Bi}_{2} \mathrm{Se}_{3}, \mathrm{Bi}_{2} \mathrm{Te}_{3}$ and $\mathrm{Sb}_{2} \mathrm{Te}_{3}$ with a single Dirac cone on the surface. Nat. Phys. 5, 438-442 (2009).

2. Hsieh, D. et al. A topological Dirac insulator in a quantum spin hall phase. Nature 452, 970-974 (2008)

3. Fu, L., Kane, C. L. \& Mele, E. J. Topological insulators in three dimensions. Phys. Rev. Lett. 98, 106803 (2007).

4. Hsieh, D. et al. A tunable topological insulator in the spin helical Dirac transport regime. Nature 460, 1101-1105 (2009).

5. Cheng, P. et al. Landau quantization of topological surface states in $\mathrm{Bi}_{2} \mathrm{Se}_{3}$. Phys. Rev. Lett. 105, 076801 (2010).

6. Analytis, J. G. et al. Two-dimensional surface state in the quantum limit of a topological insulator. Nat. Phys. 6, 960-964 (2010). 
7. Qu, D.-X., Hor, Y. S., Xiong, J., Cava, R. J. \& Ong, N. P. Quantum oscillations and hall anomaly of surface states in the topological insulator $\mathrm{Bi}_{2} \mathrm{Te}_{3}$. Science 329 , 821-824 (2010)

8. He, H.-T. et al. Impurity effect on weak antilocalization in the topolofical insulator $\mathrm{Bi}_{2} \mathrm{Te}_{3}$. Phys. Rev. Lett. 106, 166805 (2011).

9. Liu, M. et al. Crossover between weak antilocalization and weak localization in a magnetically doped topological insulator. Phys. Rev. Lett. 108, 036805 (2012).

10. Tang, H., Liang, D., Qiu, R. L. J. \& Gao, X. P. A. Two-dimensional transport-induced linear magneto-resistance in topological insulator $\mathrm{Bi}_{2} \mathrm{Se}_{3}$ nanoribbons. ACS Nano 5, 7510-7516 (2011).

11. Wang, X., Du, Y., Dou, S. \& Zhang, C. Room temperature giant and linear magnetoresistance in topological insulator $\mathrm{Bi}_{2} \mathrm{Te}_{3}$ nanosheets. Phys. Rev. Lett. 108 266806 (2012)

12. Checkelsky, J. G. et al. Quantum interference in macroscopic crystals of non metallic $\mathrm{Bi}_{2} \mathrm{Se}_{3}$. Phys. Rev. Lett. 103, 246601 (2009).

13. Checkelsky, J. G., Hor, Y. S., Cava, R. J. \& Ong, N. P. Bulk band gap and surface state conduction observed in voltage-tuned crystals of the topological insulator $\mathrm{Bi}_{2} \mathrm{Se}_{3}$. Phys. Rev. Lett. 106, 196801 (2011).

14. Analytis, J. G. et al. Bulk Fermi surface coexistence with Dirac surface state in $\mathrm{Bi}_{2} \mathrm{Se}_{3}$ : a comparison of photoemission and Shubnikov-de Haas measurements. Phys. Rev. B 81, 205407 (2010).

15. Hsieh, D. et al. Observation of time-reversal-protected single-Dirac-cone topological-insulator states in $\mathrm{Bi}_{2} \mathrm{Te}_{3}$ and $\mathrm{Sb}_{2} \mathrm{Te}_{3}$. Phys. Rev. Lett. 103, 146401 (2009).

16. Zhang, H. B. et al. Weak localization bulk state in a topological insulator $\mathrm{Bi}_{2} \mathrm{Te}_{3}$ film. Phys. Rev. B 86, 075102 (2012).

17. Peng, $H$. et al. Aharonov-Bohm interference in topological insulator nanoribbons. Nat. Mater. 9, 225-229 (2010).

18. Bianchi, M. et al. Coexistence of the topological state and a two-dimensional electron gas on the surface of $\mathrm{Bi}_{2} \mathrm{Se}_{3}$. Nat. Commun. 1, 128 (2010).

19. Veldhorst, M. et al. Magnetotransport and induced superconductivity in Bi based three-dimensional topological insulators. Phys. Status Solidi RRL 7, 26-38 (2013).

20. Culcer, D. Transport in three-dimensional topological insulators: theory and experiment. Phys. E 44, 860-884 (2012).

21. Wang, J. et al. Evidence for electron-electron interaction in topological insulator thin films. Phys. Rev. B 83, 245438 (2011).

22. Chiu, S.-P. \& Lin, J.-J. Weak antilocalization in topological insulator $\mathrm{Bi}_{2} \mathrm{Te}_{3}$ microflakes. Phys. Rev. B 87, 035122 (2013).

23. Cao, $\mathrm{H}$. et al. Controlling and distinguishing electronic transport of topological and trivial surface states in a topological insulator. arXiv:1409.3217 (2014).

24. Dey, R. et al. Strong spin-orbit coupling and Zeeman spin splitting in angle dependent magnetoresistance of $\mathrm{Bi}_{2} \mathrm{Te}_{3}$. Appl. Phys. Lett. 104, 223111 (2014).

25. Hikami, S., Larkin, A. I. \& Nagaoka, Y. Spin-orbit interaction and magnetoresistance in the two dimensional random system. Prog. Theor. Phys. 63, 707-710 (1980).

26. Dugdale, J. S. The Electrical Properties of Disordered Metals (Cambridge University Press, 1995).

27. Xiang, F.-X., Wang, X.-L., Veldhorst, M., Dou, S.-X. \& Fuhrer, M. S. Observation of topological transition of Fermi surface from a spindle torus to a torus in bulk Rashba spin-split BiTeCl. Phys. Rev. B 92, 035123 (2015).

28. Xiang, F.-X. et al. Thickness-dependent eelctronic structure in $\mathrm{WTe}_{2}$ thin films Phys. Rev. B 98, 035115 (2018).

29. Cao, H. et al. Quantized Hall effect and Shubnikov-de Haas oscillations in highly doped $\mathrm{Bi}_{2} \mathrm{Se}_{3}$ : evidence for layered transport of bulk carriers. Phys. Rev. Lett. 108, 216803 (2012).

30. Lu, H.-Z., Shi, J. \& Shen, S.-Q. Competition between weak localization and antilocalization in topological surface states. Phys. Rev. Lett. 107, 076801 (2011).

31. Gao, B. F., Gehring, P., Burghard, M. \& Kern, K. Gate-controlled linear magnetoresistance in thin $\mathrm{Bi}_{2} \mathrm{Se}_{3}$ sheets. Appl. Phys. Lett. 100, 212402 (2012).

32. $\mathrm{He}, \mathrm{H}$. et al. High-field linear magneto-resistance in topological insulator $\mathrm{Bi}_{2} \mathrm{Se}_{3}$ thin films. Appl. Phys. Lett. 100, 032105 (2012).
33. Abrikosov, A. A. Galvanomagnetic phenomena in metals in the quantum limit. J. Exp. Theor. Phys. 29, 746-753 (1969).

34. Wang, C. M. \& Lei, X. L. Linear magnetoresistance on the topological surface. Phys. Rev. B 86, 035442 (2012)

35. Zhang, D. et al. Superconducting proximity effect and possible evidence for Pear vortices in a candidate topological insulator. Phys. Rev. B 84, 165120 (2011).

36. $\mathrm{Xu}, \mathrm{R}$. et al. Large magnetoresistance in non-magnetic silver chalcogenides. Nature 390, 57-60 (1997)

37. Hu, J., Rosenbaum, T. F. \& Betts, J. B. Current jets, disorder, and linear magnetoresistance in the silver chalcogenides. Phys. Rev. Lett. 95, 186603 (2005).

38. Assaf, B. A. et al. Linear magnetoresistance in topological insulator thin films: quantum phase coherence effects at high temperatures. Appl. Phys. Lett. 102, 012102 (2013).

39. Kozlova, N. V. et al. Linear magnetoresistance due to multiple-electron scattering by low-mobility islands in an inhomogeneous conductor. Nat. Commun. 3, 1097 (2012).

40. Khouri, T. et al. Linear magnetoresistance in a quasifree two-dimensional electron gas in an ultrahigh mobility GaAs quantum well. Phys. Rev. Lett. 117, 256601 (2016).

41. $\mathrm{Xu}, \mathrm{X}$. et al. Directional field-induced metallization of quasi-one-dimensional $\mathrm{Li}_{0.9} \mathrm{Mo}_{6} \mathrm{O}_{17}$. Phys. Rev. Lett. 102, 206602 (2009).

42. Graf, D. et al. Suppression of a charge-density-wave ground state in high magnetic fields: spin and orbital mechanisms. Phys. Rev. B 69, 125113 (2004).

43. Wang, L.-X. et al. Zeeman effect on surface electron transport in topological insulator $\mathrm{Bi}_{2} \mathrm{Se}_{3}$ nanoribbons. Nanoscale 7, 16687-16694 (2015).

44. Wang, J. et al. Anomalous anisotropic magnetoresistance in topological insulator films. Nano Res. 5, 739-746 (2012).

45. Scanlon, D. O. et al. Controlling bulk conductivity in topological insulators: key role of anti-site defects. Adv. Mater. 24, 2154-2158 (2012).

46. Arnold, F. et al. Negative magnetoresistance without well-defined chirality in the Weyl semimetal TaP. Nat. Commun. 7, 11615 (2016).

47. Huang, S.-M. et al. A Weyl Fermion semimetal with surface Fermi arcs in the transition metal monopnictide TaAs class. Nat. Commun. 6, 7373 (2015)

48. Liu, Z. K. et al. Discovery of a three-dimensional topological Dirac semimetal, $\mathrm{Na}_{3} \mathrm{Bi}$. Science 343, 864-867 (2014).

49. $\mathrm{Li}, \mathrm{H}$. et al. Negative magnetoresistance in Dirac semimetal $\mathrm{Cd}_{3} \mathrm{As}_{2}$. Nat. Commun. 7, 10301 (2016).

50. Goswami, P., Pixley, J. H. \& Das Sarma, S. Axial anomaly and longitudinal mag netoresistance of a generic three-dimensional metal. Phys. Rev. B 92, 075205 (2015).

51. Argyres, P. N. \& Adams, E. N. Longitudinal magnetoresistance in the quantum limit. Phys. Rev. 104, 900-908 (1956).

52. ViolBarbosa, C. E. et al. Direct observation of band bending in the topological insulator $\mathrm{Bi}_{2} \mathrm{Se}_{3}$. Phys. Rev. B 88, 195128 (2013).

53. Tang, $H$. et al. Thermoelectric characterization of individual bismuth selenide topological insulator nanoribbons. Nanoscale 7, 6683-6690 (2015).

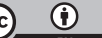

Open Access This article is licensed under a Creative Commons Attribution 4.0 International License, which permits use, sharing, adaptation, distribution and reproduction in any medium or format, as long as you give appropriate credit to the original author(s) and the source, provide a link to the Creative Commons license, and indicate if changes were made. The images or other third party material in this article are included in the article's Creative Commons license, unless indicated otherwise in a credit line to the material. If material is not included in the article's Creative Commons license and your intended use is not permitted by statutory regulation or exceeds the permitted use, you will need to obtain permission directly from the copyright holder. To view a copy of this license, visit http://creativecommons. org/licenses/by/4.0/.

(c) The Author(s) 2019 